\title{
Selected Topics Relating to Functional MRI Study of the Brain
}

\author{
Seiji Ogawa and Yul-Wan Sung \\ Kansei Fukushi Research Institute, Tohoku Fukushi University, Sendai, Japan \\ (Received for publication on December 8, 2018) \\ (Revised for publication on February 25, 2019) \\ (Accepted for publication on March 11, 2019) \\ (Published online in advance on April 11, 2019)
}

\begin{abstract}
The authors selected some interesting current topics among many in the field of functional MRI (fMRI) of the brain. The selection was based on authours' immediate interests in exploring these aspects further; the topics are presented and discussed along with their perspectives. If progress can be made in these areas, it would be very advantageous to the field of brain research. The topics are (I) Detectable MRI signals in response to functional activity of the brain, including the current status of neurocurrent MRI; (II) Vascular-dependent and vascular-independent MRI signals, leading to the distinction of functional and structural MRI; (III) Functional specificity and functional connectivity of local sites, including differences between task-fMRI and resting state fMRI; (IV) Functional networks: an example of application to assessing the vocational aptitude test by fMRI; (V) Neural oscillation relevant to the formation of fMRI signals and of networks; (VI) Upgrading fMRI to "information-content-reflecting" fMRI, discussed as one of the prospects of near-future fMRI. (DOI: 10.2302/kjm.2018-0011-OA; Keio J Med 68 (4) : 73-86, December 2019)
\end{abstract}

Keywords: fMRI, neurocurrent, resting sate fMRI, functional network, information content

\section{Introduction}

Currently, one focus of brain science is connecting the function of the brain to human society by finding representations of human behavior (including intelligential/ mental or emotional characters and neuro-disorders) in functional architectures of the brain. One reason for this trend is that many researchers consider that current knowledge of how the brain works in terms of its functional activity has expanded enough to be utilized. Functional MRI (fMRI) has played a major role in brain research and has become a routine way of checking the function of the brain in many settings. fMRI is based on the vascular response to functional activation of the brain; it is not a direct measure of the electrochemical activity of the neural system, unlike other investigative approaches that measure brain waves (i.e., electroencephalography, or EEG) or brain magnetic waves (i.e., magnetoencephalography, or MEG). Moreover, fMRI has a major disad- vantage in that it lacks the capability to follow the fast dynamics of neural events because of its slow response time, which is in the order of seconds. Nonetheless, fMRI has the ability, as its main feature, to pinpoint locations of functioning sites in the brain at good spatial resolution. EEG, MEG, and other noninvasive methodologies lack this capability.

The most common fMRI method uses blood oxygenation level-dependent (BOLD) contrast. This image contrast is produced by magnetic field disturbances inside and outside of blood vessels which contain paramagnetic deoxyhemoglobin. Such field disturbances are generated by the magnetic susceptibility difference between these blood vessels and the surrounding tissue (Fig. 1A left). The magnetic field disturbance at the surface of a blood vessel is of the order of $10^{-7}$ that of the homogeneous main magnetic field of MRI. The field disturbance contributes to the value of the $\mathrm{T} 2 *$ or the $\mathrm{T} 2$ decay constant of the MRI signal. 
A Baseline state

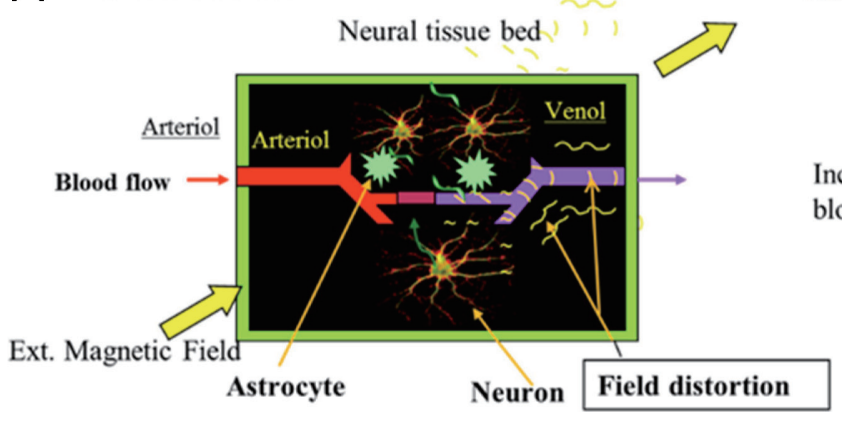

BOLD effect

\section{Activated state}

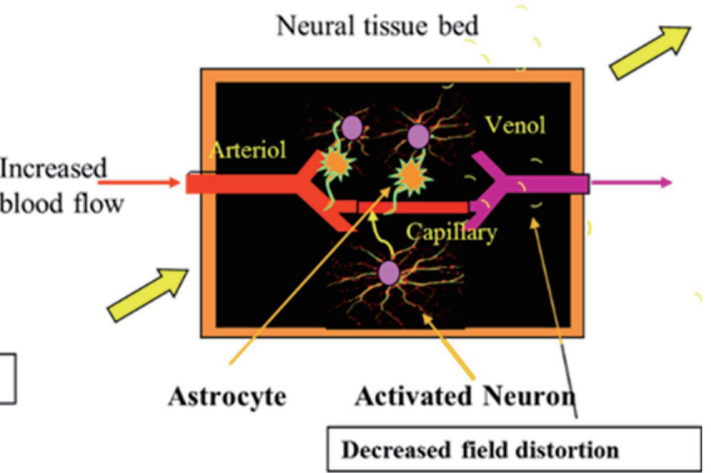

BOLD Effect with neuro-activation

B

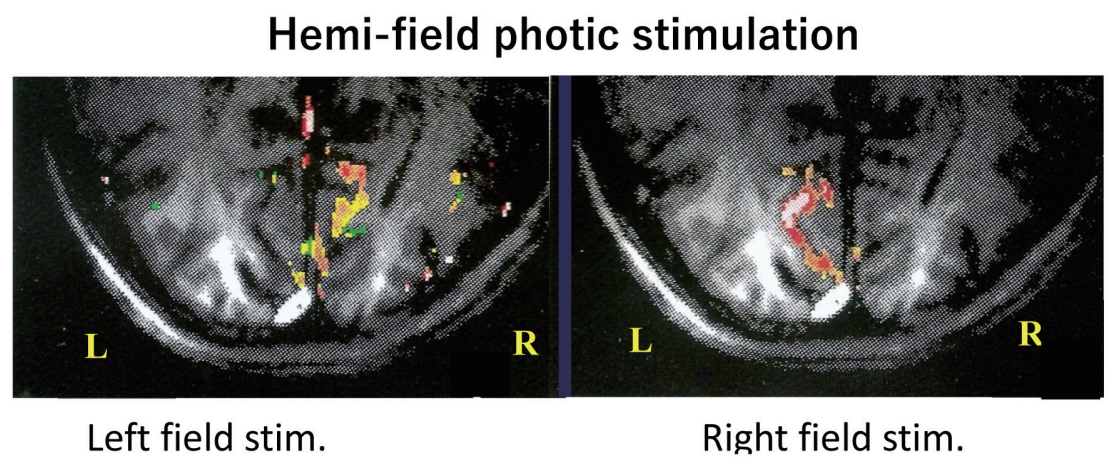

Fig. 1 (A) Schematic showing the effect of neural activation on BOLD contrast. The left panel shows the basal state, and the right panel shows the neuronal activation state. Red vessels (arteriols) indicate oxygenated blood and purple vessels represent the presence of deoxyhemoglobin in the blood. Pink color vessels in the left drawing indicate the decrease of the level of deoxyhemoglobin with neuro activation. Ext, external. (B) BOLD fMRI observation of V1 activation at contralateral hemispheres by hemifield photic stimulation. Reprocessed from the data in Ogawa et al. 1992. ${ }^{1}$

It has been known for 100 years that neural activation in the brain induces an increase of local cerebral blood flow (CBF). Furthermore, the increased CBF boosts the oxygen supply to an extent greater than that required to meet local metabolic needs. This results in a decreased concentration of deoxyhemoglobin in the capillaries and venous blood vessels. This mechanism by which neural activation mediates BOLD contrast in fMRI images ${ }^{1,2}$ is shown schematically in Fig. 1.

Fig. 1A depicts the field distortions generated near and inside blood vessels that contain deoxyhemoglobin. The presence of such field distortion induces faster signal decay (larger $\mathrm{R}_{2}{ }^{*}$ ) in that area (darker areas in the cortex shown in Fig. 1B) and generates the image contrast. In the neuro-activated state, the excess blood flow induced by neurovascular coupling makes the venous blood reddish from the original purple color (Fig. 1A) seen at the baseline state by decreasing deoxyhemoglobin concentrations. The resulting reduced field disturbance leads to a signal increase that produces the neuro-induced change in BOLD contrast. The neurovascular coupling process is briefly described in the following Section I titled Vascular Response.

One early observation of neural activation of visual areas in the human brain is shown in the BOLD-based fMRI in Fig. 1B in the case of left or right hemi-field photo stimulation. Brain activation appeared along the cortical areas where the signal changes are indicated by red color in the image. These cortical areas are away from the fovea area where signals generated by center-field photic stimuli would appear.

With the tight coupling between neural activation and blood vessel dilation, this local change of MRI signal is a reliable indicator of the local changes in neural activity. Furthermore, the noninvasive nature of the method, a major advantage of MRI, allows healthy volunteers to participate as subjects in fMRI-based research without raising ethical concerns. This fact has resulted in expansion of the field of fMRI application.

However, the slow response time of fMRI is a disadvantage, as is the dependence of the BOLD signal on multiple parameters such as CBF, oxygen consumption, 
and the amount of deoxyhemoglobin as well as the vascular structure at the location of the signal. These factors make detailed quantitative analysis using fMRI difficult, and we have to be content with regarding the signal as a reporter of the functional response of the brain. Despite these disadvantages, fMRI signal acquisition is straightforward because there is no need to preset the proton spins into a specialized condition to acquire the intended signal.

There have been tremendous developments and advances in the field of fMRI since its inception in the early $1990 \mathrm{~s}^{1,2}$ Analyses of functional neural networks in the resting state as well as in a state of task-induced activation are leading to an understanding of human psychological and medical characteristics expressed as networks in the human brain. ${ }^{3}$ The artificial intelligence (AI) approach of neural networks based on Deep Learning has been applied to improve the image quality obtained from fMRI data and to understand how the brain encodes its inputs and how that information can be decoded from brain image data. ${ }^{4}$ Nonetheless, efforts to clarify the basics of in vivo MRI measurement in terms of the relation between structure and function of the brain have also been a focus of the MRI community. In particular, research has been carried out to try to distinguish the vascular signal from the tissue signal, including attempts to produce vasculardependent and vascular-independent images. ${ }^{5,6}$ Technical developments, such as very fast acquisition methods and submillimeter high-spatial resolution imaging, ${ }^{7}$ are bringing fMRI and its in vivo data closer to the recent neuroscience approach of studying the function of neuronal assemblies beyond the activity of a single or a few neurons.

In this article, we select several topics of interest and discuss them according to our research perspectives. These topics are: (I) Detectable MRI signals in response to functional activity of the brain, (II) Vascular-independent and vascular-dependent MRI signals, (III) Functional specificity of local sites and their functional connectivity, (IV) Functional networks: an example of application, (V) Neural oscillation is relevant to the formation of fMRI signals and networks, and (VI) Upgrading fMRI to information-content-reflecting fMRI.

\section{(I) Detectable MRI Signals in Response to Functional Activity of the Brain}

\section{Neurocurrent MRI}

Thirty years ago, it was commonly understood within the MRI research community that brain function (essentially electrochemical reactions) was not detectable by MRI. For in vivo measurement, this still seems true today, despite the advantages of MRI (such as noninvasiveness and simultaneous acquisition of structural information) being extremely attractive in brain research. There are various neurocurrents that could generate local magnetic fields capable of influencing the behavior of proton spins central to MRI. Examples of these include magnetic field induced by neuronal action potentials, by currents of axonal pulse conduction, and by post-synaptic currents in the dendritic bed of functioning neuronal assemblies. None of these seem to induce detectable signal changes in in vivo MRI because of the low signal-to-noise ratio. Moreover, the generated magnetic fields may not be suitable for MRI detection because of their dynamics, spatial distribution, and orientation.

The magnetic field generated by the action potential (AP) of a single neuron or by currents supporting axonal pulse propagation has a bipolar character, reversing direction in approximately a millisecond, as shown in Fig. 2, reproduced from Barry et al. 2016. ${ }^{9}$ These dynamic magnetic field changes, apparently induced by $\mathrm{Na}^{+}$and $\mathrm{K}^{+}$currents, were measured by a closely located optomagnetic sensor based on nitrogen-vacancy color centers in diamond crystal. Such opto-magnetic probes, when miniaturized, will be able to decode the axonal pulse trains related to known animal behaviors, allowing us to begin understanding the language the brain uses.

There are likely abundant micro-scale post-synaptic currents in the dendritic bed of an assembly of neurons coherently activated to perform functional processing. The synaptic currents running in parallel fibers and penetrating through the multiple layers of the cortex should be good candidates for in vivo MRI detection. MEG, which measures extremely small extracranial neuro-magnetic fields (i.e., the far field), is thought to have its source in these parallel-fiber synaptic currents. ${ }^{10}$ In contrast, EEG measures the population average of local field potentials (LFPs) with which synaptic currents are associated.

Recently, Sundaram et al. ${ }^{11}$ reported the detection of neurocurrents in excised but intact turtle cerebelli, which could tolerate hypoxia and remain physiologically functional. The advantage of this preparation comes from the absence of vascular signals, heart or respiration noise, and tissue motion, thereby making high spatial resolution imaging possible. On electrical stimulation of intact turtle cerebellum in the presence of an inhibitor, only the slow neuronal activity mediated by metabotropic glutamate receptors produced an LFP. This LFP was measured both by grids of electrodes and by high-spatial-resolution "functional" MRI $\left(0.3 \mathrm{~mm}^{3}\right)$. Sundaram et al. mapped the LFP associated with the neural activation and estimated the current dipole distribution in space. The MRI signal phase shift peaked at $0.3^{\circ}$ concurrently with the LFP, as shown in Fig. 3. However, the magnitude of the MRI signal change was very small, indicating that the effective magnetic field to which the proton spins were exposed was quite homogeneous within the individual voxels of the area. The decay time of the phase shift was quite close to the slow decay of the LFP of this particular neuronal activity. The current dipole distribution was estimated 
A

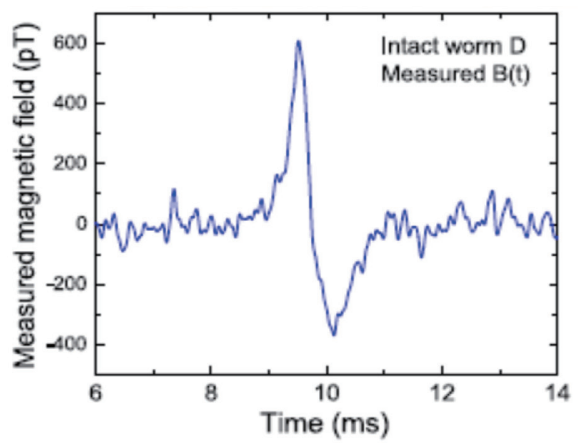

B

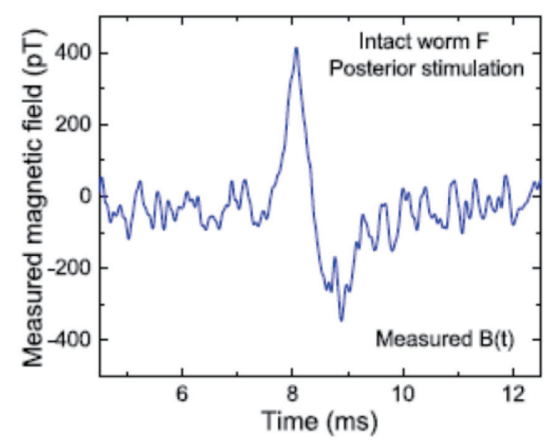

Fig. 2 Magnetic fields produced by currents with action potential and axonal pulse conduction in marine fanworms. Measurements were made using the optically detected magnetic resonance of electron spin in negative vacancy diamond crystal. Reproduced with permission from Barry et al., ${ }^{9}$ Fig. 3B and Fig. 4E. (A) Recorded time trace of single-neuron AP magnetic field from a live intact specimen of Myxicola infundibulum for $\mathrm{N}_{\mathrm{avg}}=1650$ events. (B) Recorded time trace of propagating AP magnetic field in the same species by posterior stimulation of the axon of an intact live specimen for $\mathrm{N}_{\mathrm{avg}}=1650$ events.

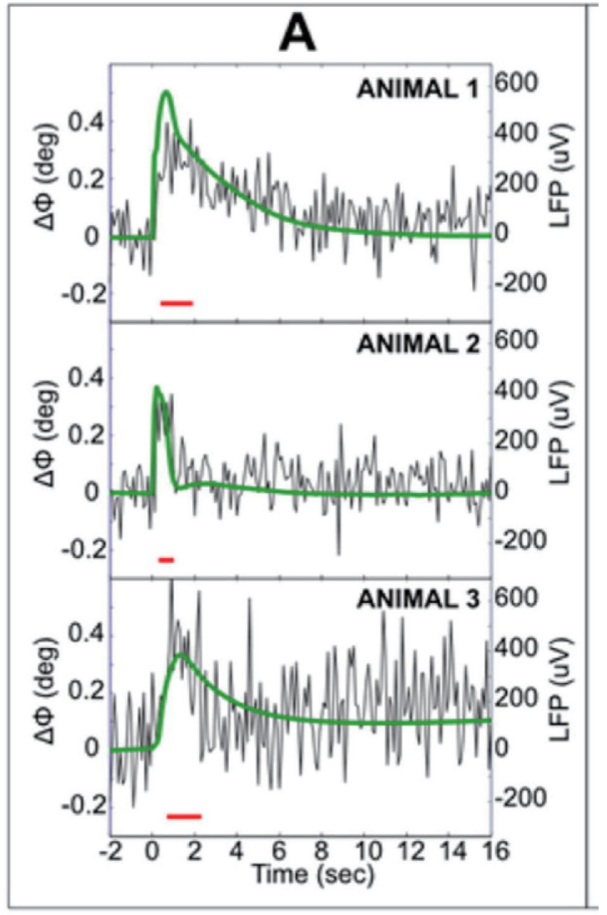

Fig. 3 The neurocurrent-induced MRI signal phase was shown to be coincidental to the LFP temporal pattern (smooth lines) from slow neuronal activity mediated by metabotropic glutamate receptors in turtle cerebellum. The small bars at the bottom of the traces indicate the stimulation periods. The phase shift was the average of 160 trials together with the sum of 37,49 , and 17 voxels for samples from three animals. Reproduced with permission from Sundaram et al., 2016, 11 Fig. 11A. from the MRI phase-shift map, and the maximum dipole strength, Q, at peak was about $0.1 \mathrm{nA} \cdot \mathrm{m}$ in a $0.33 \times 0.33$ $\mathrm{mm}^{2}$ pixel. The estimated two dipole maps of LFP and fMRI were spatially similar.

Based on the above results of Sundaram et al., it is worth considering what would happen in cases where LFP decay is fast, e.g., within a few tens of milliseconds, as seen with usual evoked potentials. The signal height would be determined by the signal decay rate balancing the signal increase rate. The signal would reach a plateau within the period of the stimulation or pass a peak after the stimulation has ceased. If the population-averaged magnetic field related to the population-averaged LFP does generate the phase shift in the MRI signal, the phase shift would decrease along with the decay of LFP. This means that the amount of phase shift would be much smaller than that in the case of very slow decay of LFP.

The results discussed above illustrate what to expect for the effects of neurocurrent on the MRI signal of intact animal samples. Consequently, the magnetic field associated with neurocurrents should be detectable as an average with a large number of repetitions, provided that the various sources of noise are suppressed, the neuro-system is stable during repetitions, and that other type of functional signals are separated. The importance of this work is the simultaneous measurement of LFP and the coincidental MRI phase shift. Because there are many other similar signals the origin of which are unknown and which may not be related to neuronal activity, the above approach to check the coincidence with LFP is currently the only way to identify neurocurrents using MRI. ${ }^{12}$ From these results in ex vivo preparations, one can hope that someday it may be possible to use MR to detect in vivo neuronal electrical reactions. The advantage of such measurements would 


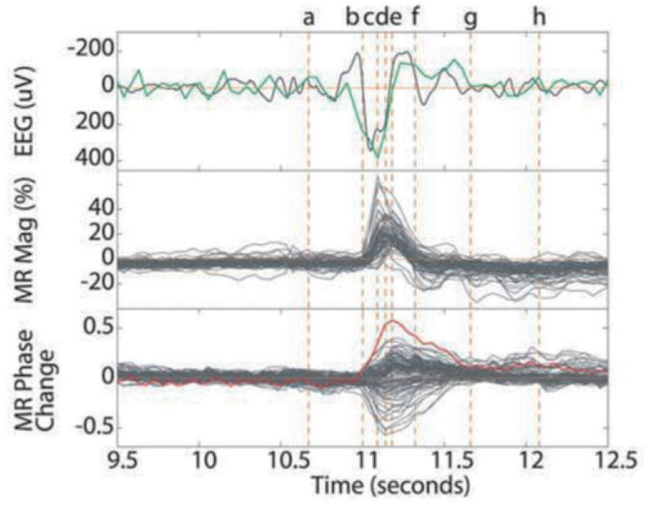

Fig. 4 Tight temporal correspondence was evident between the interictal spike seen on EEG (black plot, top panel) and the percent change in MRI magnitude (middle panel) and the phase change in radians (bottom panel). Reproduced from Sundaram et al., ${ }^{14}$ Fig. 3.

be in determining quantitative physical values, such as the current dipole strength, and elucidating the dynamics of the event that needs to be stable for repetition to be successful. Other than the dynamics, the information deduced from a current-induced MRI signal would be related to the occurrence of a neuro-event at a specific site. The characteristics of the event that the neurocurrent indicates may be similar to those of LFP (measured by EEG) or MEG signals, or even similar to fMRI responses in the sense that the signals do not provide the information content of the specific processing function of the specific brain region.

\section{Epileptic state MRI signal}

Considering the difficulty in observing neurocurrentinduced MRI signals in the human brain, reports of MRI signal changes associated with epileptiform spikes ${ }^{13}$ are surprising. During interictal discharges, MRI magnitude and phase signal changes (at a repetition time of image acquisition TR $=47 \mathrm{~ms}$ ) appeared with easily observable high values. These changes were in concert with the temporal dynamic variation of EEG signals. The appearance and the decay rate of the MRI magnitude and phase signals were delayed from the LFP peaks by less than a second, as shown in Fig. 4, reproduced from Sundaram et al. 2010. ${ }^{13}$ The signal decay is much faster than usual BOLD signals. The possibility that bulk head motion was the cause of the such MRI signal changes was excluded by the subsequent report by the same authors; ${ }^{14}$ nonetheless, the motion of local brain tissue water by some neuro-mechanical coupling mechanism remains a possibility. However, as discussed by the authors, ${ }^{13}$ these large MRI signal changes (a maximum of $75 \%$ relative to the
MRI signal of quiet periods) were observed at the time of interictal spikes on EEG that had unexceptional heights. This observation of such large MRI signal changes is very difficult to comprehend other than as a local image shift at a location with a large gap in MRI signal, such as at the edge of an image or next to cerebrospinal fluid-rich voxels. The Lorentz force even in a large magnetic field $\mathrm{B}_{0}$ would not produce such a strong effect by the neurocurrent expected from the observed ordinary spike height of EEG.

\section{Vascular responses}

Another way to detect functional activity of the brain using MRI is based on the vascular/metabolic responses to neural activation. One such approach is BOLD-fMRI, as mention in the Introduction, and it has been highly popular since its genesis in the early $1990 \mathrm{~s}^{1,2,15}$; it is currently routinely used for the study of brain function. Changes in the cerebral blood volume (CBV) are known to be well linked to neural activation. CBV changes offer better spatial specificity and a shorter signal rise time (by about 1s) than BOLD signals. Moreover, CBV signals do not have a signal component from large surface venous vessels. In 2003, vascular space occupancy (VASO)-dependent fMRI was proposed by a group at Johns Hopkins University to measure CBV changes. ${ }^{16}$ The inversion recovery signal of tissue water at the null time point of the blood water signal can be sensitive to blood volume changes resulting from the decrease of the tissue water content in an imaging voxel caused by an increase in blood vessel volume. The CBV changes in VASO measurements likely include those at arterioles, capillaries, and venous vessels. Recent advances of MRI technology and increased understanding of the signal change process have turned this method into an emerging fMRI tool to investigate the neuroscience aspects of neuro-imaging. ${ }^{17,18}$

The key factor of such indirect measurements of neural activation through vascular responses is the tightness of the neurovascular coupling. The coupling mechanism has been attributed not to the action of $\mathrm{Ca}^{2+}$ in astrocytes, but to that of $\mathrm{K}^{+}$released by glutamate binding to astrocytes and to neurons near the synaptic junction whose activity releases excess glutamate. The released $\mathrm{K}^{+}$makes the smooth muscle of the blood vessel surface dilate and also binds to the endothelium, the inner surface of blood vessels, to induce vessel dilation. ${ }^{19-22}$ With this neuro-vascular coupling, we can expect function-induced changes in BOLD signal.

The slow response (in seconds) to neural events of some $100 \mathrm{~ms}$ or shorter is the main disadvantage of BOLD fMRI and other vascular-response approaches to fMRI. However, the slow decay rate of vascular signals increases the sensitivity of the signal detection. The slow decay 
72

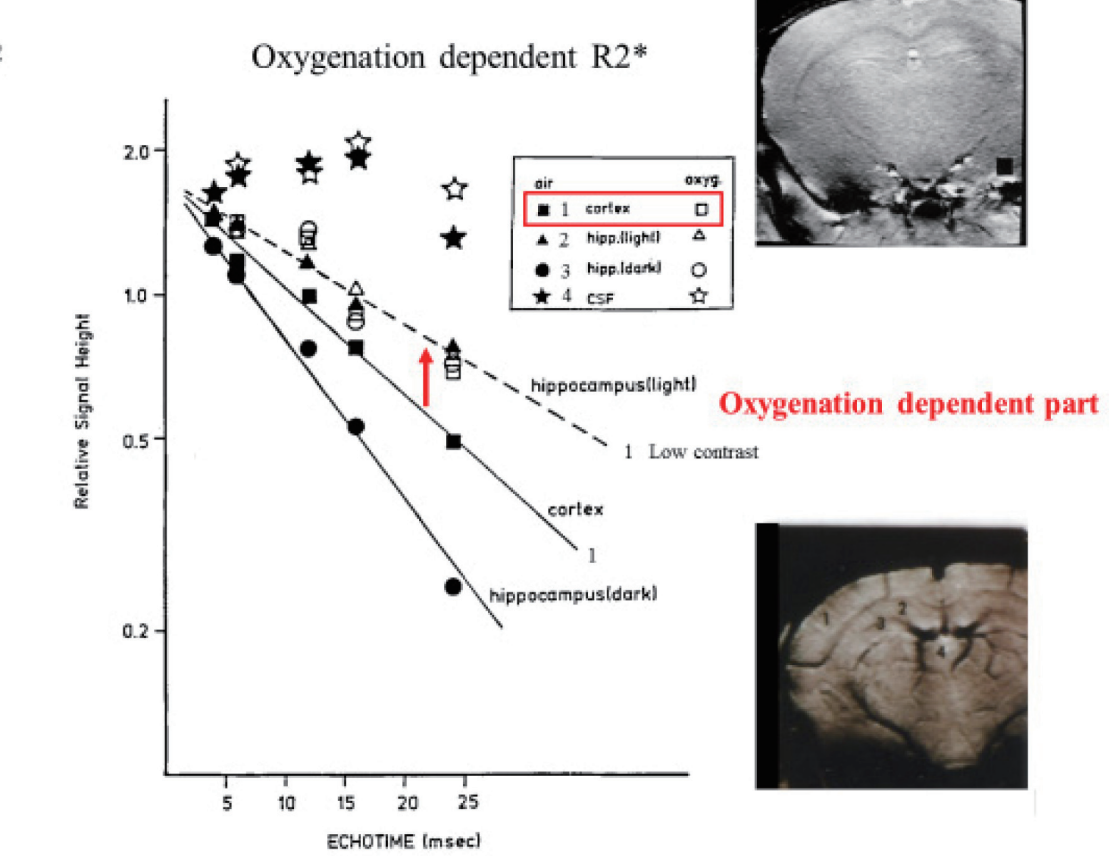

Fig. 5 Signal decay as a function of echo time in gradient echo brain images of an anesthetized mouse. The solid symbols are for the image signals of the brain of a mouse breathing normal air (bottom image), and the open symbols are for the image signals of the highly oxygenated brain (top image) of the same mouse. The change in the signal intensity between the two conditions is not due to functional activation. CSF, cerebrospinal fluid. The figure includes some text added to the original in Ogawa et al. $1990 .{ }^{15}$

time allows BOLD signals to accumulate until the signal production matches the decay, either reaching a plateau or passing the signal response peak. The main reasons behind the popularity of fMRI in brain research are the tight coupling with neural events and the relatively high sensitivity to functional activity.

\section{(II) Vascular-dependent and Vascular-independent MRI Signals}

BOLD contrast, which is based on vascular-dependent signal changes, reflects one aspect, among many, of the brain functions that have been measured by fMRI. However, vascular-independent signals could be sensitive to functional and physiological brain states, in addition to the anatomical tissue specific-contrast of the structural image. The MRI signal S at an echo time TE can be expressed as follows:

$$
\begin{aligned}
& \mathrm{S}=\mathrm{S}_{0} \exp \left(-\mathrm{R}_{2} * \cdot \mathrm{TE}\right)=\mathrm{S}_{1}+\mathrm{S}_{2} \\
& =\mathrm{S}_{01} \exp \left\{-\left(\mathrm{R}_{2} * \text { tissue }\right) \cdot \mathrm{TE}\right\} \\
& +\mathrm{S}_{02} \exp \left\{-\left(\mathrm{R}_{2} * \text { tissue }+\mathrm{R}_{2} * \text { vasc }\right) \cdot \mathrm{TE}\right\}
\end{aligned}
$$

where the $\mathrm{S}_{01}$ term corresponds to the signal in the area not influenced by the vascular response, and the $\mathrm{S}_{02}$ term indicates the area covered by the response caused by vascular effects and the TE dependence with two combined relaxation rates.

When there is neural activation, the fractional signal change will be:

$$
\begin{aligned}
& \Delta \mathrm{S} / \mathrm{S} \sim\left(-\Delta \mathrm{R}_{2} * \text { tissue } \cdot \mathrm{TE}\right) \cdot \mathrm{S}_{1} / \mathrm{S} \\
& +\left(-\left(\Delta \mathrm{R}_{2} * \text { tissue }+\Delta \mathrm{R}_{2} * \text { vasc }\right) \cdot \mathrm{TE}\right) \cdot \mathrm{S}_{2} / \mathrm{S} \\
& \text { Assuming } \Delta \mathrm{R}_{2} * \text { tissue }=0, \Delta \mathrm{S} / \mathrm{S} \sim\left(-\Delta \mathrm{R}_{2} * \text { vasc } \cdot \mathrm{TE}\right) \\
& \mathrm{S}_{2} / \mathrm{S}
\end{aligned}
$$

where $S_{2} / S$ is the signal fraction of the area of the vascular effect coverage in the voxel.

In an early report on $\mathrm{R}_{2}{ }^{*}$ in the mouse brain, oxygenation-dependent signal changes were observed, as shown in Fig. 5, as a function of TE. ${ }^{13}$ In the highly oxygenated brain, the signal contrast largely decreased, as in Fig. 5 (upper image), and the residual signal should closely represent the vascular-independent term, largely free from the vascular term. The vascular-dependent term includes functional as well as other physiological effects that could modify the oxygenation and venous blood volume.

Recent efforts have been directed at extracting the vascular term from the quantitative BOLD response ${ }^{5}$ or at producing theoretical estimates of the BOLD signal ${ }^{6}$ and subtracting it from the total $\mathrm{R}_{2} *$ term. What remained should be the tissue term, although it likely contains the part of the vascular dependent term that responds to non- 
functional but physiological condition because the above estimates of vascular dependent term are usually based on the functional change of BOLD signal. In this approach, the estimate of the vascular term must be very accurate, otherwise the functional response of the vascular independent tissue term could be due to the residual from the vascular dependent term. If this approach is successful, the " $\mathrm{R}_{2}{ }^{*}$ tissue" term can be isolated and its sensitivity to functional and physiological responses can be checked. ${ }^{23}$ The rest of the signal contains contrasts of the anatomical structure. Provided that the anatomical structure image has sufficiently strong contrasts of several types, the anatomical structure map could indicate the presence of various components characterizing the capabilities of various biological functions in the tissue area. It is hoped that, at ultra-high magnetic fields such as $11.7 \mathrm{~T}$ or higher, MRI using the above approach will provide new insight into the working of the human brain.

Another approach to obtain the $\mathrm{R}_{2}{ }^{*}$ tissue term mentioned above would be to use the resting state MRI signals that have been attributed to the basal state of the brain. Although such time series signals are quite noisy and hard to pinpoint in vascular terms, it is known that BOLD type signal changes representing resting state brain function do exist. ${ }^{24}$ Such functional resting state signals occur at frequencies lower than $0.1 \mathrm{~Hz}$ in the time series. The resting state signal during the period when the functional signals do not appear must be related to the basal state of the neural system and should include the non-functional vascular term. This vascular term as a BOLD effect has the same coverage over the tissue as the functional vascular term, but it is likely controlled either by a mechanism without neuronal involvement or by one with neuronal involvement in a nonfunctional role. The effect of $\mathrm{CO}_{2}$ intake, which induces an increase in $\mathrm{CBF}$, must appear as a signal variation in this term. This term should be related to the basic metabolic state which influence the deoxyhemoglobin content in the blood. Therefor the term would be very informative about not only the autoregulation of CBF but also the basal energy metabolism of the brain, which is known to be highly aerobic with an oxygen/glucose consumption ratio of near 5.5. The oxygen consumption during functional neural activation does not reach this high oxygen consumption. The vascular response seen in the BOLD effect at the basal state would be interesting to explore. ${ }^{25}$ If the basal vascular term could be removed from the basal state signal, the result would be the real basal tissue term. The question of how to achieve this separation needs a further understanding of the phenomena involved.

\section{(III) Functional Specificity of Local Sites and Their Functional Connectivity}

Knowledge about the specificity of functional sites is important to understand how the brain works. However, such specificities have been determined only through estimates from postmortem lesion studies and from the expected input to a site dictated by various related neuroimaging tasks. Often the specificity is of a category that is very general. Determination of such specificity from noninvasive measurements is very difficult, especially at higher-order functioning sites. All site specificity determinations are estimates from indirect information on the way the site works; the reason for this is that the contents of the output information cannot so far be measured noninvasively.

\section{Functional modules}

There may be various functional submodules with varying specificity within the neuro-assembly cluster of a one-centimeter-sized site or a voxel of $(3 \mathrm{~mm})^{3}$. Functional imaging at a submillimeter spatial resolution would allow such submodules to be distinguished by their input characteristics. For processing a particular functional task, a combination of relevant modules would be activated. Using fMRI at a low spatial resolution, the presence of certain modules can be tested by observing the event-related responses to a pair of stimulation pulses of the same or different tasks occurring within a short interval (e.g., in tens to hundreds of milliseconds). When the same task is used in a pair of stimuli, the fMRI response of the second stimulus is highly suppressed. However, for task pairs that are different, this suppression does not occur, indicating that the two stimuli activate different modules comprising independent neural assemblies. ${ }^{26}$ When the above-described refractory response is observed with partial suppression for non-identical two tasks, the results would be interpreted in such a way that some functional modules in the cluster site are interacting each other. Furthermore, varying the inter-stimulus interval would allow measurement of the extent to which the tasks interact with or remain independent from each other.

However, the refractory response, which can be used to distinguish different tasks in a pair of stimuli, could loose this character by some top-down control, such as the expectation of pair pulses with a different task combination. Such cases often appear in adaptation paradigms. ${ }^{27}$ In such situations, the activation of higher-order sites, such as those of the attention circuit, becomes observable, indicating that higher-order sites impose top-down control on the lower functional sites. ${ }^{28}$

A highly developed language capability is unique to humans, and the language processing network of the human brain has been extensively studied. ${ }^{29}$ Among the areas involved in language processing, the Broca's area of Brodmann's areas (BA) 44 and 45 corresponds to the area assigned 100 years ago by Paul Broca as the site for word generation. Based on clinical and postmortem studies on his patient's brain, Broca claimed that it was an example 
of brain function localization. Currently, BA 44 is known to be involved in syntax (grammar) processing, whereas BA 45 is specialized in semantic processing.

Several studies have looked into second language acquisition ${ }^{30}$ among speakers of European languages that have a similar language basis. However, Sakai referred to his studies on fMRI responses to short "grammar" tests in second language L2 (English) given to mature Japanese subjects who learnt language L2 when they were young. ${ }^{31}$ The two languages [the first language L1 (Japanese) and the second language L2 (English)] differ significantly in structure and grammar. Region BA44 was activated by L2 tasks, overlapping with the activation area resulting from similar tests in L1. For young Japanese subjects who were proficient in L2, the activation by the L2 test was less intense and in a limited area of BA 44, indicating either language area plasticity or lower functional group recruitment in the area after good training. Moreover, the same study reported that similar tests in volunteers who were not exposed to L2 learning did not activate the site during the English grammar test.

It is interesting to note that the brain exerted two types of functional facility (L1 and L2 grammar check) within the same area. This raises several questions, such as is there a mosaic-like placement of functional modules for the two languages. Furthermore, is there any level of neuron sharing between the two functions or are there instant wiring changes among neurons to perform functions related to L1 and L2? To detect grammar violations in processed sentences, there may be some storage of templates with L1 and/or L2 grammatical patterns in the area. Such questions could be answered if the spatial distribution of the detailed specificities in the modular arrangement of the functioning area could be determined and close interactions among such modules could be found.

\section{Functional connectivity}

The functional connectivity of a site in the brain to various remote sites has been determined using the temporal correlation between activation signals in resting state fMRI images. These images contain many intrinsic, functional brain activations without any particular tasks to perform. ${ }^{32,33}$ This connectivity must include those from all functional varieties within a cluster assembly, where spontaneous and coherent activations of all modules are presumably present. The connectivity from such a cluster to remote sites would form an infra-structure among brain sites, similar to that shown by axonal bundle connections that have been observed with diffusion tensor imaging/fiber tracking. In task-fMRI data, where only a limited subset of modules is used, some connectivity observed in resting state fMRI may be inhibited or excluded. For example, in the case of primary motor areas of the left and right hemispheres, this situation is observable. In the resting state, both M1 sites are highly correlated, ${ }^{32}$ although the motor task needed to move the right hand activates only the M1 of the contralateral left hemisphere, and not the ipsilateral M1. The activation of some sub-modules within a site by a specific task is only a part of the resting state functional activation. However, its connectivity to other sites must be specific to the functional processing of the task-related input.

\section{(IV) Functional Networks: An Example of Applica- tion}

Resting state BOLD activation, which appears as signal fluctuations at very low frequencies (below $0.1 \mathrm{~Hz}$ ), seems to be a fundamental property of the brain. ${ }^{33}$ The functional connectivity map of the brain may contain many functional networks. Therefore, resting state signal correlation analysis has great potential to detect functional brain states. Such analysis may also identify networks determining human sensory and mental characteristics, and also networks newly modified by learning (plasticity) or disturbed by central nervous system disease. ${ }^{34}$

One example we have been working on is to find biomarkers of various capabilities expressed in brain networks for vocational aptitude tests. ${ }^{35}$ We hope that these biomarkers will be useful for students to investigate their vocational fitness in various aptitude categories. The algorithm to identify such neural networks, which comprises 19 General Aptitude Test battery categories, is shown in Fig. 6. ${ }^{35}$

Three lines of information were gathered and are indicated in Fig. 6A as lines (I), (II), and (III). Line (I) shows the psychological 19-category test scores of 112 undergraduate college students as the volunteer subjects, line (II) shows anatomical structural information of 112 subjects with 272 predetermined regions of interests (ROIs) in the standard brain, and line (III) shows functional information collection and processing. Figure 6B shows the processing in line (III): (a) for partial data of the resting state time-series of one of the 112 students, (b) for predetermined ROI's in the standard brain, (c) for each functional correlation matrix of 272 dimensions for 112 subjects, and (d) for processing to find the functional networks for each test category by correlating the 112 test scores with the 112 functional correlation parameters (vij, $\mathrm{j}=1-112$ ) of $\mathrm{i}$-th element in the 112 matrices of these 112 subjects, covering all functional correlation parameters $\mathrm{i}=1-\mathrm{N}, \mathrm{N}=272 \times 271 / 2$.

Figure 7 presents an example of such functional networks for the category Design \& Art. This network is composed of 14 nodes (ROIs), among which the middle occipital gyrus, the fusiform gyrus, and the parahippocampal gyrus are known to be involved in visual object and visuospatial context information processing, whereas the amygdala, brain stem, and temporal pole are known to be involved in social and emotional processing. Within 


\section{A A procedure to find functional networks for job categories}

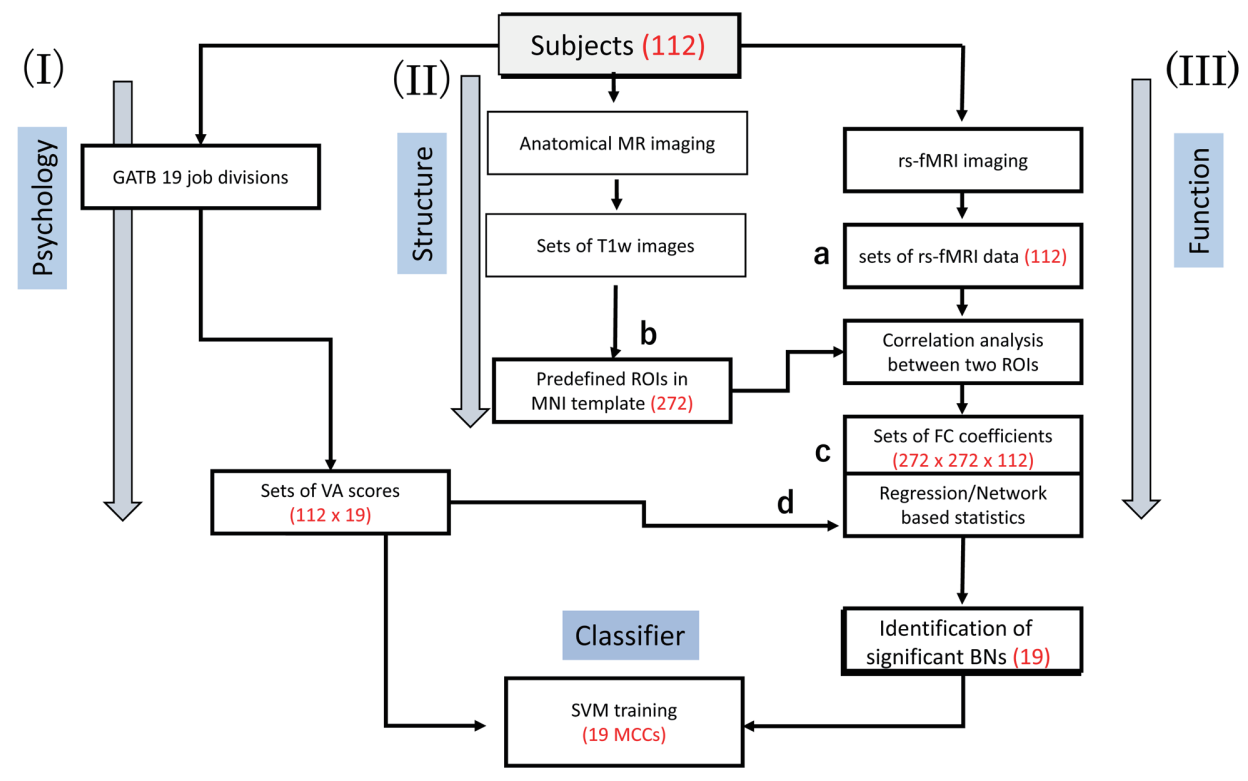

B

(a)

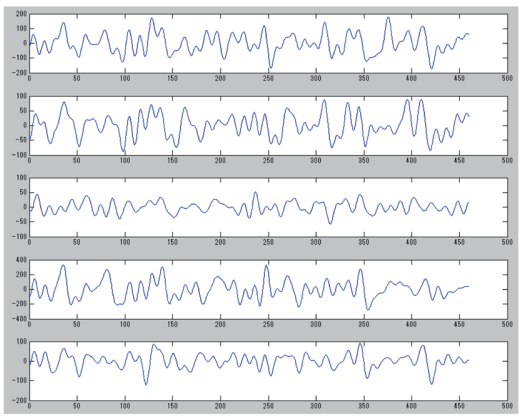

(b)

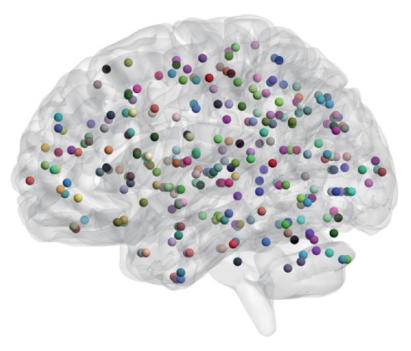

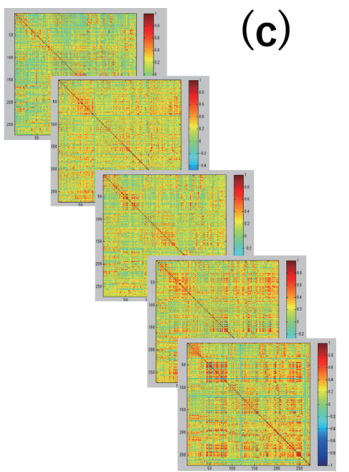

(d)

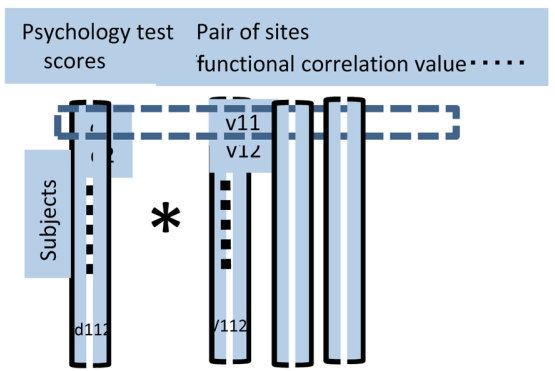

Fig. 6 (A) Schematic showing the procedure to find functional networks for 19 job categories from (I) psychological test scores, (II) anatomical image acquisition and predetermined ROI, (III) functional data acquisition and analyses. GATB, general aptitude test battery; VA, Vocational Aptitude; MNI, Montreal Neurological Institute; MCC, multi-class classifier; BN, brain network. (B) Details at a, b, c, and d in Fig. 6A. (a) Resting state time series data at 272 ROIs for individual subjects; (b) the 272 ROIs; (c) the correlation matrix for 112 subjects; and (d) correlating the psychological test scores of 112 individuals with their functional connectivity parameters of 112 matrices to find a network for a testing category (see text for the detail). 

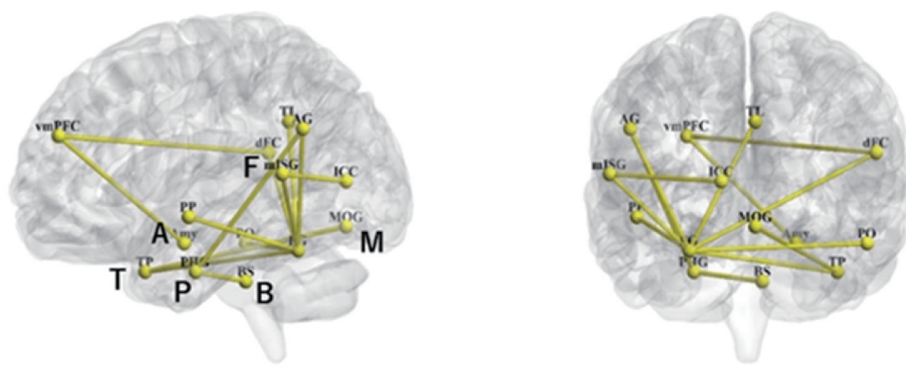

Fig. 7 The functional network for the category of Design \& Art. MOG, M, middle occipital gyrus; FG, F, fusiform gyrus, PHG, P, parahippocampal gyrus; Amy, A, amygdala; BS, B, brain stem; and TP, T, temporal pole.

the network, the functional roles of each brain area and the correlation parameters among the nodes describe the network's characteristic for the vocational category.

As shown at the bottom of Fig. 6A, classifiers were built for the 19-category tests to checks a new subject's aptitudes. This was done using a support vector machine algorithm with 112 subjects' psychology test scores and the correlation parameters of the functional network elements observed in the matrices of these 112 subjects. To evaluate the reliability of these classifiers, the classifiers were applied to resting state fMRI data and the aptitude test scores of 19 categories for new subjects. Both the classifier grading and the psychological test scores were categorized in three levels: high, medium, or low. Compared with the psychological tests scores, the reliability was superior to a $30.3 \%$ chance level, and the average of the 19 categories was about $60 \%$.

To apply the abovementioned approach to "real-life" tests of student fitness, many improvements are needed. For a reliable individual level evaluation, repeated fMRI measurements of the same subject may be useful to secure the accuracy of aptitude prediction. The dynamic connectivity variation, when existent, could be averaged. Further reliability improvement is expected through enhancement of the signal-to-noise ratio of fMRI data, ${ }^{36}$ as well as by reducing uncertainties between fMRI and behavioral data. One way to remove such uncertainties is to measure fMRI data from professionals/experts of each vocational field, by which the determination of training end-points may be possible. To date, we have experienced difficulty in enrolling such experts in a substantial number for any of the fields.

\section{(V) Neural Oscillation is Relevant to fMRI Signals and Networks}

The following paragraph contains a quote from the review by Buzsaki and Draguhn published in Science in $2004 .{ }^{37}$

"Mammalian cortical neurons form behavior-dependent oscillating networks of various sizes, which span five orders of magnitude in frequency. These oscillations are phylogenetically preserved, suggesting that they are functionally relevant. Recent findings indicate that network oscillations bias input selection, temporally link neurons into assemblies, and facilitate synaptic plasticity, mechanisms that cooperatively support temporal representation and long-term consolidation of information."

The oscillations mentioned above include the delta $(0.5-4 \mathrm{~Hz})$, theta $(4-7 \mathrm{~Hz})$, alpha $(8-13 \mathrm{~Hz})$, beta $(13-$ $30 \mathrm{~Hz})$, and gamma $(>30 \mathrm{~Hz})$ oscillations. Gamma oscillations, however, may not be an element of general information transfer within a macro-network, but likely relate to local functional activity. ${ }^{38}$ At a local level, the BOLD signal may reflect the coordination of the neuronal firing mediated by gamma-frequency oscillations in postsynaptic potentials. These oscillations can facilitate communication between neurons in the local area by ensuring that spiking occurs at the peak excitability moment of the oscillatory local field potential. ${ }^{39}$

To understand the roles of these various oscillations, it would be relevant to find how these low- and high-frequency oscillations work in concert, coordinating neural activity into the whole-brain functional networks indicated by fMRI. ${ }^{40}$ Multimodal measurements, such as EEG and fMRI, should lead to the identification of functionally connected sites with similar low frequency oscillations.

It would be beneficial if low frequency oscillations could be directly identified in the fMRI signal. However, considering the slow dynamics of BOLD signals, this does not seem possible, except for resting state slow oscillations of less than $0.1 \mathrm{~Hz}$. Nevertheless, whether BOLD or other functional signals carry some characteristics of the paradigm-driven fast dynamics in the fMRI slow response has not been explored. If this is possible, then the low frequency matching with the functionally connected sites could be directly measured with some temporal phase relation, demonstrating real functional connectivity. Although this is a big "if," it is worth exploring, because the requirement of very fast fMRI signal acquisition with good signal-to-noise ratios is within the 
reach of current methodology.

\section{(VI) Upgrading fMRI to Information-content-re- flecting fMRI}

BOLD signals show the location of functional activation but do not inform us about the content of the group activity processing. BOLD signals tend to correspond closely to LFPs, with a minor contribution from neuronal firing, although the information processing by the group corresponds to the collection of firing activities of the participating neurons. The usefulness of fMRI lies in its ability to indicate noninvasively the locations and relative strength of the functional activation of the sites that the brain mobilizes to perform a given task. The functional specificity of these sites is roughly known from the accumulated knowledge of lesion studies and from recent neuro-imaging results. Using that knowledge as a basis, fMRI results of functional site locations are useful to understand what kind of action the brain is taking. This location finding, without knowing the information content of the group activity, is common to the noninvasive methods used in brain research, such as MEG and EEG, which have the additional ability to observe fast activity dynamics. A considerable part of the success of brainmachine interfaces (BMIs) may well be attributed to the information usage of brain locations activated to perform motor tasks. ${ }^{41}$

The information content of the activity of a group of neurons while processing their input must be changing along the dynamically proceeding activity and is impossible to follow until the group finds the result for the purpose of the activity. The only opportunity and place to see to what the group activity leads relate to the activity of the output neurons. In the lamellar structure of the cortex, layers V and VI are known to transmit the results of upper layer processing. If it became possible to detect the activity of the output neurons of these layers, we could have some handle on understanding the information of the output from a bundle of inputs to the site (i.e., the result of the functional site activity). In other words, we could clarify the specificities of the site and how they vary with inputs. The knowledge of such behavioral and image information would enrich the understanding of brain function to a great extent, because we would also be getting close to the phenomena of information transfer for communication between sites by the specific functional connectivity.

Recently, successful studies that used MRI and fMRI to analyze cortical layers at submillimeter spatial resolution have been reported. ${ }^{42,43}$ In a special edition of NeuroImage in $2018,{ }^{7}$ Kemper et al. ${ }^{44}$ described a strategy for high-resolution fMRI data analysis. It is difficult to cover the whole brain successfully because of the long imaging time and intra-image head motion. Some problems with the post-imaging correction of head motion may result in blurring of the details of high-resolution images. In situ motional correction would be advantageous to overcome this shortcoming. The surface macrovessel effect on fMRI signals from lower layers has been addressed. ${ }^{45}$ VASO measurements may not encounter this problem, because the susceptibility effect on $\mathrm{R}_{2} *$ does not extent in space due to the extremely short TE (echo time for image formation) which prevents intravoxel phase dispersion to contribute to $\mathrm{R}_{2}{ }^{*}$, contrary to the case of regular gradient echo imaging.

Localized fMRI to investigate a particular cortical area would be a strategy to study layer activity. This approach would have the advantages of shorter acquisition times and a choice of coordinates to visualize the layers properly. Koretsky et al. showed details of layered signal characters in their line-scanned image analysis. ${ }^{46}$

If deep layer measurement of the human brain can be established in high spatial resolution, it would be advantageous to find a way to extract such signals from relatively low-resolution images that could be acquired more easily. We are planning an attempt to compare two images that differ in image position by one or two layer thicknesses and extract the bottom layers from the others.

The most important point in estimating the information content of output neuron activity using fMRI signals is the neurovascular coupling at the deep layers. Potassium ion $\left(\mathrm{K}^{+}\right)$release from astrocytes and neurons upon the binding of glutamate from synaptic activity has been claimed to have a role in neurovascular coupling. ${ }^{21}$ The released $\mathrm{K}^{+}$is bound to the outer smooth muscle layer of blood vessels and also to the endothelium of the inner wall of blood vessels, thereby inducing vessel dilation. This vascular response is then related to the LFP because the synaptic activity is the starter of the coupling reaction. With this coupling, however, BOLD signals do not reflect the information content of the output activity of pyramidal cells in the deep layers.

In interesting optogenetic studies by Kahn et al., 8,47 it was shown that in optogenetically manipulated mice that have photo-controllable channels (to activate the cells) in pyramidal cells in layer V, AP activity (cell firing) resulting from photo-stimulation was confined in layer V. However, the LFP spread to the upper layers to some extent, as detected using electrical physiology measurements $^{8}$ (Fig. 8A). By varying the pattern of the induced firing trains, the responding BOLD signals were shown to have fairly good sensitivity to the variation (Fig. 8B), indicating the possibility that neurovascular coupling at layer $\mathrm{V}$ is mainly through cell firing, rather than through LFP or synaptic activity. Furthermore, Scott and Murphy ${ }^{48}$ reported that the vascular response at a depth of $250 \mu \mathrm{m}$ to sensory stimulation was highly reduced by antagonists (CNQX/MK801) to the glutamate binding site; however, the vascular response to the activation of layer V pyramidal cells via photo-control did not change with the presence or absence of antagonists (Fig. 8C). The 

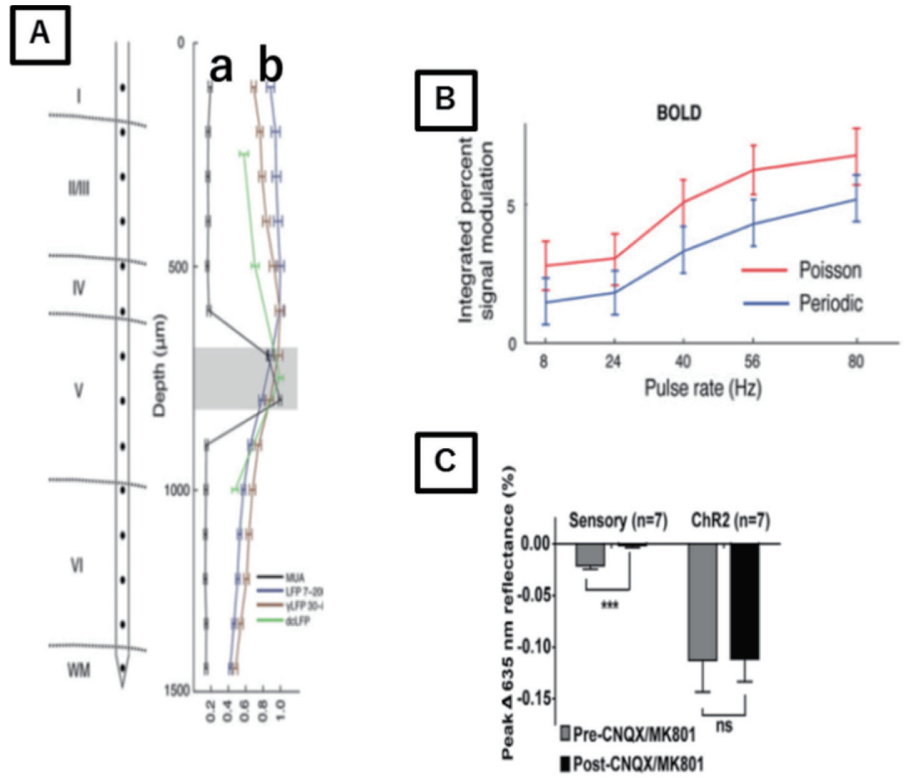

Fig. 8 Electrical and vascular responses to stimulation of optogenetically manipulated pyramidal cells of layer V in mice. (A) Electrode measurements of the pyramidal cell activation in terms of (a) firing activity and (b) LFP. (B) Photo-stimulation pattern variation influence on the BOLD response. Reproduced from with permission Kahn et al., ${ }^{8}$ Fig. 4A and 4C. (C) Blood volume change signals for a depth of $250 \mu \mathrm{m}$. Data are shown for the presence and absence of an antagonist for glutamate binding to astrocytes and neurons [ketamine $(20 \mathrm{mg} / \mathrm{ml}) / x y l a z i n e ~(1 \mathrm{mg} / \mathrm{ml})]$ in anesthetized mice. Reproduced from with permission Scott et al., ${ }^{48}$ Fig. $3 \mathbf{E}$.

presence of such antagonists inhibits the neurovascular coupling related to synaptic activity, and the residual vascular response could be caused by cell firing activity. ${ }^{48}$ In the study of Scott and Murphy, the vascular response was measured at the upper layers at a depth of $250 \mu \mathrm{m}$ from the surface. The reported results of the two groups described above are conflicting. ${ }^{8,48} \mathrm{Kahn}$ et al. showed that cell firing activity was limited to layer $\mathrm{V}$ only, the layer where the photo-activation occurred. In the report of Scott and Murphy, the vascular response at the upper layers was not related to synaptic activity even when the photo-stimulation was at a deep layer. Some detailed comparison of the relevant experimental conditions is required.

If this vascular response to neural firing comprises most of the response at deep layers and is the same in the cortical layers of the human brain, then it might be possible to monitor the information content of the functioning site's output. The sensitivity of the MRI response and the representability of the pyramidal cell firing in the area of the observation are the key factors. When the input to the area is varied by selecting the paradigm contents in task-fMRI, a change in the vascular response might be observed in a way that can be understood and interpreted, with a good probability that some information content can be elucidated. Furthermore, the variation in the specificity of the functioning modules in the area could be observed. There may be cases of no variation in the vascular response despite differing inputs within a category. This means that either the site (or the observing module) is category-specific, or the site has a filter that extracts only specific information from the various inputs to it, making that the specificity of the site.

A better understanding of the brain's functional specificity, especially at higher-order brain functional sites, would allow us to learn its functional architecture with less reliance on speculative explanation. The current stage of understanding the brain means that the specificities of functioning sites are mostly estimates with rather broad coverage. After the assignment of specific functional roles to sites throughout the brain is accomplished, interpreting functional networks of various categories would become more plausible, because we will have a fairly good idea on the transferred information from one site to another in a network. We may also gain insights into efficient information processing in the way the brain proceeds to solve a given task. Such knowledge may be useful for improving the performance of AI algorithms, which so far have been using very many uniform operational amplifiers with nonspecific highly-multiple connections. With a better understanding of how the brain works, brain science will be able to interact forcefully with the real world of human society.

There are many other topics of interests in the field of brain research. The topics covered here were selected based on our immediate interests in exploring them fur- 
ther. Other researchers may have already investigated these topics and, if that is the case, we would be very happy to learn about the results. We expect that fMRI will continue to progress further and will furnish greater understanding of how the brain works.

\section{Conflicts of Interest}

The authors have no conflicts of interest related to this report.

\section{References}

1. Ogawa S, Tank DW, Menon R, Ellermann JM, Kim SG, Merkle $\mathrm{H}$, Ugurbil K: Intrinsic signal changes accompanying sensory stimulation: functional brain mapping with magnetic resonance imaging. Proc Natl Acad Sci USA 1992; 89: 5951-5955. PMID:1631079, DOI:10.1073/pnas.89.13.5951

2. Ogawa S, Lee TM, Kay AR, Tank DW: Brain magnetic resonance imaging with contrast dependent on blood oxygenation. Proc Natl Acad Sci USA 1990; 87: 9868-9872.

3. Glasser MF, Smith SM, Marcus DS, Andersson JL, Auerbach EJ, Behrens TE, Coalson TS, Harms MP, Jenkinson M, Moeller S, Robinson EC, Sotiropoulos SN, Xu J, Yacoub E, Ugurbil K, Van Essen DC: The Human Connectome Project's neuroimaging approach. Nat Neurosci 2016; 19: 1175-1187. PMID:27571196, DOI:10.1038/nn.4361

4. Naselaris T, Kay KN, Nishimoto S, Gallant JL: Encoding and decoding in fMRI. Neuroimage 2011; 56: 400-410.

5. Blockley NP, Griffeth VE, Simon AB, Dubowitz DJ, Buxton RB: Calibrating the BOLD response without administering gases: comparison of hypercapnia calibration with calibration using an asymmetric spin echo. Neuroimage 2015; 104: 423-429. PMID:25451475, DOI:10.1016/j.neuroimage.2014.09.061

6. Ulrich X, Yablonskiy DA: Separation of cellular and BOLD contributions to T2* signal relaxation. Magn Reson Med 2016; 75: 606-615. PMID:25754288, DOI:10.1002/mrm.25610

7. Yacoub E, Wald LL: Pushing the spatio-temporal limits of MRI and fMRI. Neuroimage 2018; 164: 1-3. PMID:29254519, DOI:10.1016/j.neuroimage.2017.11.034

8. Kahn I, Knoblich U, Desai M, Bernstein J, Graybiel AM, Boyden ES, Buckner RL, Moore CI: Optogenetic drive of neocortical pyramidal neurons generates fMRI signals that are correlated with spiking activity. Brain Res 2013; 1511: 33-45. PMID:23523914, DOI:10.1016/j.brainres.2013.03.011

9. Barry JF, Turner MJ, Schloss JM, Glenn DR, Song Y, Lukin MD, Park H, Walsworth RL: Optical magnetic detection of singleneuron action potentials using quantum defects in diamond. Proc Natl Acad Sci USA 2016; 113: 14133-14138. PMID:27911765, DOI:10.1073/pnas.1601513113

10. Okada YC, Nicholson C: Magnetic evoked field associated with transcortical currents in turtle cerebellum. Biophys J 1988; 53: 723-731. PMID:3390518, DOI:10.1016/S0006-3495(88)83153-9

11. Sundaram P, Nummenmaa A, Wells W, Orbach D, Orringer D, Mulkern R, Okada Y: Direct neural current imaging in an intact cerebellum with magnetic resonance imaging. Neuroimage 2016; 132: 477-490. PMID:26899788, DOI:10.1016/j.neuroimage.2016.01.059

12. Petridou N, Plenz D, Silva AC, Loew M, Bodurka J, Bandettini PA: Direct magnetic resonance detection of neuronal electrical activity. Proc Natl Acad Sci USA 2006; 103: 16015-16020. PMID:17038505, DOI:10.1073/pnas.0603219103

13. Sundaram P, Wells WM, Mulkern RV, Bubrick EJ, Bromfield EB, Münch M, Orbach DB: Fast human brain magnetic resonance re- sponses associated with epileptiform spikes. Magn Reson Med 2010; 64: 1728-1738. PMID:20806355, DOI:10.1002/mrm.22561

14. Sundaram P, Mulkern RV, Wells WM, Triantafyllou C, Loddenkemper T, Bubrick EJ, Orbach DB: An empirical investigation of motion effects in eMRI of interictal epileptiform spikes. Magn Reson Imaging 2011; 29: 1401-1409. PMID:21550748, DOI:10.1016/j.mri.2011.03.007

15. Ogawa S, Lee TM, Nayak AS, Glynn P: Oxygenation-sensitive contrast in magnetic resonance image of rodent brain at high magnetic fields. Magn Reson Med 1990; 14: 68-78. PMID:2161986, DOI: $10.1002 / \mathrm{mrm} .1910140108$

16. Lu H, Golay X, Pekar JJ, van Zijl PC: Functional magnetic resonance imaging based on changes in vascular space occupancy. Magn Reson Med 2003; 50: 263-274. PMID:12876702, DOI:10.1002/mrm.10519

17. Lu H, van Zijl PC: A review of the development of VascularSpace-Occupancy (VASO) fMRI. Neuroimage 2012; 62: 736742. PMID:22245650, DOI:10.1016/j.neuroimage.2012.01.013

18. Huber L, Tse DH, Wiggins CJ, Uludağ K, Kashyap S, Jangraw DC, Bandettini PA, Poser BA, Ivanov D: Ultra-high resolution blood volume fMRI and BOLD fMRI in humans at $9.4 \mathrm{~T}$ : capabilities and challenges. Neuroimage 2018; 178: 769-779. PMID:29890330, DOI:10.1016/j.neuroimage.2018.06.025

19. Filosa JA, Bonev AD, Straub SV, Meredith AL, Wilkerson MK, Aldrich RW, Nelson MT: Local potassium signaling couples neuronal activity to vasodilation in the brain. Nat Neurosci 2006; 9: 1397-1403. PMID:17013381, DOI:10.1038/nn1779

20. Girouard H, Bonev AD, Hannah RM, Meredith A, Aldrich RW, Nelson MT: Astrocytic endfoot $\mathrm{Ca}^{2+}$ and BK channels determine both arteriolar dilation and constriction. Proc Natl Acad Sci USA 2010; 107: 3811-3816. PMID:20133576, DOI:10.1073/ pnas.0914722107

21. Longden TA, Hill-Eubanks DC, Nelson MT: Ion channel networks in the control of cerebral blood flow. J Cereb Blood Flow Metab 2016; 36: 492-512. PMID:26661232, DOI:10.1177/0271678X15616138

22. Masamoto K, Unekawa M, Watanabe T, Toriumi H, Takuwa H, Kawaguchi H, Kanno I, Matsui K, Tanaka KF, Tomita Y, Suzuki $\mathrm{N}$ : Unveiling astrocytic control of cerebral blood flow with optogenetics. Sci Rep 2015; 5: 11455. PMID:26076820, DOI:10.1038/ srep11455

23. Griffeth VE, Perthen JE, Buxton RB: Prospects for quantitative fMRI: Investigating the effects of caffeine on baseline oxygen metabolism and the response to a visual stimulus in humans. Neuroimage 2011; 57: 809-816. PMID:21586328, DOI:10.1016/j. neuroimage.2011.04.064

24. Peltier SJ, Noll DC: T2* Dependence of Low FrequencyFunctional Connectivity. NeuroImage 2002;16, 985-992.PMID:12202086, DOI:10.1006/nimg.2002.1141

25. Hyder F, Rothman DL: Quantitative fMRI and oxidative neuroenergetics. Neuroimage 2012; 62: 985-994. PMID:22542993, DOI:10.1016/j.neuroimage.2012.04.027

26. Sung YW, Ogawa S: Using FMRI for elucidating dynamic interactions. Methods Mol Biol 2009; 489: 243-254. PMID:18839095, DOI:10.1007/978-1-59745-543-5 11

27. Summerfield C, Trittschuh EH, Monti JM, Mesulam MM, Egner $\mathrm{T}$ : Neural repetition suppression reflects fulfilled perceptual expectations. Nat Neurosci 2008; 11: 1004-1006. PMID:19160497, DOI:10.1038/nn.2163

28. Choi US, Sung YW, Ogawa S: Steady-state and dynamic network modes for perceptual expectation. Sci Rep 2017; 7: 40626.

29. Friederici AD: Language in Our Brain: The Origin of a Uniquely Human Capacity, MIT Press 2017.

30. Friederici AD: Language in Our Brain: The Origin of a Uniquely Human Capacity, MIT Press 2017 pp149-154. 
31. Sakai KL: Language acquisition and brain development. Science 2005; 310: 815-819. PMID:16272114, DOI:10.1126/science. 1113530

32. Biswal B, Zerrin Yetkin F, Haughton VM, Hyde JS: Functional connectivity in the motor cortex of resting human brain using echo-planar MRI. Magn Reson Med 1995; 34: 537-541. PMID:8524021, DOI:10.1002/mrm.1910340409

33. Raichle ME: A paradigm shift in functional brain imaging. J Neurosci 2009; 29: 12729-12734. PMID:19828783, DOI:10.1523/ JNEUROSCI.4366-09.2009

34. Khazaee A, Ebrahimzadeh A, Babajani-Feremi A: Application of advanced machine learning methods on resting-state fMRI network for identification of mild cognitive impairment and Alzheimer's disease. Brain Imaging Behav 2016; 10: 799-817. PMID:26363784, DOI:10.1007/s11682-015-9448-7

35. Sung YW, Kawachi Y, Choi US, Kang D, Abe C, Otomo Y, Ogawa $\mathrm{S}$ : Estimation of vocational aptitudes using functional brain networks. Hum Brain Mapp 2018; 39: 3636-3651. PMID:29717529, DOI:10.1002/hbm. 24200

36. de Zwart JA, Gelderen P, Fukunaga M, Duyn JH: Reducing correlated noise in fMRI data. Magn Reson Med 2008; 59: 939-945. PMID:18383291, DOI:10.1002/mrm.21507

37. Buzsáki G, Draguhn A: Neuronal oscillations in cortical networks. Science 2004; 304: 1926-1929. PMID:15218136, DOI:10.1126/science. 1099745

38. Hermes D, Miller KJ, Wandell BA, Winawer J: Gamma oscillations in visual cortex: the stimulus matters. Trends Cogn Sci 2015; 19: 57-58. PMID:25575448, DOI:10.1016/j.tics.2014.12.009

39. Fries P: A mechanism for cognitive dynamics: neuronal communication through neuronal coherence. Trends Cogn Sci 2005; 9: 474-480. PMID:16150631, DOI:10.1016/j.tics.2005.08.011

40. Whitman JC, Ward LM, Woodward TS: Patterns of cortical oscillations organize neural activity into whole-brain functional networks evident in the fMRI BOLD signal. Front Human Neurosci 2013; 7: 80-84.
41. Kawato M: Decoding brain information: the future BMI will open. Asahi Shimbun Publishing Co. 2010.

42. Chaimow D, Yacoub E, Uğurbil K, Shmuel A: Spatial specificity of the functional MRI blood oxygenation response relative to neuronal activity. Neuroimage 2018; 164: 32-47. PMID:28882632, DOI:10.1016/j.neuroimage.2017.08.077

43. De Martino F, Yacoub E, Kemper V, Moerel M, Uludag K, DeWeerd P, Ugurbil K, Goebel R, Formisano E: The impact of ultrahigh field MRI on cognitive and computational neuroimaging. Neuroimage 2018; 168: 366-382.

44. Kemper VG, De Martino F, Emmerling TC, Yacoub E, Goebel $\mathrm{R}$ : High resolution data analysis strategies for mesoscale human functional MRI at 7 and 9.4 T. Neuroimage 2018; 164: 48-58. PMID:28416453, DOI:10.1016/j.neuroimage.2017.03.058

45. Yu X, Glen D, Wang S, Dodd S, Hirano Y, Saad Z, Reynolds R, Silva AC, Koretsky AP: Direct imaging of macrovascular and microvascular contributions to BOLD fMRI in layers IV-V of the rat whisker-barrel cortex. Neuroimage 2012; 59: 1451-1460. PMID:21851857, DOI:10.1016/j.neuroimage.2011.08.001

46. Yu X, Qian C, Chen DY, Dodd SJ, Koretsky AP: Deciphering laminar-specific neural inputs with line-scanning fMRI. Nature Methods 2014; 11: 55-58.

47. Kahn I, Desai M, Knoblich U, Bernstein J, Henninger M, Graybiel AM, Boyden ES, Buckner RL, Moore CI: Characterization of the functional MRI response temporal linearity via optical control of neocortical pyramidal neurons. J Neurosci 2011; 31: 1508615091. PMID:22016542, DOI:10.1523/JNEUROSCI.0007-11.2011

48. Scott NA, Murphy TH: Hemodynamic responses evoked by neuronal stimulation via channelrhodopsin-2 can be independent of intracortical glutamatergic synaptic transmission. PLoS One 2012; 7: e29859. PMID:22253807, DOI:10.1371/journal. pone.0029859 